\title{
Difference sets and the primes
}

\author{
by
}

Imre Z. Ruzsa (Budapest) and Tom Sanders (Cambridge)

1. Introduction. In this paper we prove the following theorem.

TheOREm 1.1. Suppose that $N$ is an integer and $A \subset\{1, \ldots, N\}$ is such that the difference between any two elements of $A$ is never one less than a prime. Then $|A|=O(N \exp (-c \sqrt[4]{\log N}))$ for some absolute $c>0$.

The first explicit upper bounds for $|A|$ are due to Sárközy [Sár78] who showed, under the same hypotheses, that

$$
|A|=O(N \exp (-(2+o(1)) \log \log \log N)) .
$$

Recently, in [Luc07], Lucier improved Sárközy's argument using the formidable methods of Pintz, Steiger and Szemerédi from [PSS88]. Indeed, he showed that

$$
|A|=O(N \exp (-\omega(N) \log \log \log N)),
$$

for some function $\omega(N)$ tending to infinity as $N$ tends to infinity $\left({ }^{1}\right)$.

Complementing these results, the first author, in [Ruz84], showed that the bound on $|A|$ cannot be too small. Specifically, that paper contains the following theorem.

Theorem 1.2. For any integer $N$, there is a set $A \subset\{1, \ldots, N\}$ with $|A| \geq \exp ((\log 2 / 2+o(1)) \log N / \log \log N)$ such that the difference between any two elements of $A$ is never one less than a prime.

The gap between the upper and lower bounds is, of course, incredibly large, but even assuming the Generalized Riemann Hypothesis, which would simplify our argument considerably, we could only get an upper bound of the shape

$$
|A|=O(N \exp (-c \sqrt{\log N})),
$$

2000 Mathematics Subject Classification: Primary 11L07.

Key words and phrases: difference sets, shifted primes, Fourier transform, exponential sums.

$\left({ }^{1}\right)$ In fact he gets $\omega(N) \sim c \log \log \log \log \log N$ for some absolute $c>0$. 
for some absolute $c>0$. Thus we are led to the following natural question, asked by the first author in [Ruz82], and with which we close our introduction.

Question. Assuming the Generalized Riemann Hypothesis, can one achieve a bound of the shape $|A|=O\left(N^{1-c+o(1)}\right)$, for some absolute $c>0$, in Theorem 1.1?

2. An outline of the paper. The driving ingredient behind the proof of Theorem 1.1 is an energy increment argument which would be made significantly easier if we had good estimates for the distribution of primes in arithmetic progressions; the main work of the paper comes from having to deal with the so-called exceptional zeros of $L$-functions. Our proof, then, begins in $\S 4$, by recalling some of the tools necessary for dealing with such zeros.

The argument splits roughly into two cases. If there is no exceptional zero then we have relatively good estimates for the primes in progressions and the energy increment method has no complications.

If there is an exceptional zero then, by averaging, we pass to a progression of common difference equal to the modulus of the character corresponding to the exceptional zero. We then conduct the energy increment argument relative to this progression.

The two cases have separate major arcs estimates for the Fourier transform of the primes; these are proved in $\S 5$. The minor arcs are then dealt with in the usual, unified, manner in $\S 6$.

It is possible to do away with the above bifurcation if one uses a carefully weighted version of the primes. However, doing this adds complications to the minor arcs estimates. Of course, once one has put the work in to get these minor arc estimates the method can be more easily transferred to other situations.

Having completed the basic Fourier estimates in $\S \S 4,5 \& 6$, we prove some energy increment results in $\S 7$ which are used in $\S 8$ to prove the main "iteration" lemma. Finally, we complete the proof of Theorem 1.1 in $\S 9$.

3. Notation. Our main tool is the Fourier transform on $\mathbb{Z}$. We identify the dual group of $\mathbb{Z}$ with $\mathbb{T}$ via the function $e(\theta):=\exp (2 \pi i \theta)$. Specifically, every additive character $\gamma: \mathbb{Z} \rightarrow \mathbb{C}$ has the form $\gamma(x):=e(\theta x)$ for some $\theta \in \mathbb{T}$. Now, we define the Fourier transform $\widehat{\imath}: \ell^{1}(\mathbb{Z}) \rightarrow L^{\infty}(\mathbb{T})$ to be the map which takes $f \in \ell^{1}(\mathbb{Z})$ to

$$
\widehat{f}(\theta):=\sum_{x \in \mathbb{Z}} f(x) \overline{e(x \theta)},
$$


and similarly convolution to be the map $*: \ell^{1}(\mathbb{Z}) \times \ell^{1}(\mathbb{Z}) \rightarrow \ell^{1}(\mathbb{Z})$ which takes $f, g \in \ell^{1}(\mathbb{Z})$ to

$$
f * g(x)=\sum_{x \in \mathbb{Z}} f\left(x^{\prime}\right) g\left(x-x^{\prime}\right) .
$$

As usual with the Fourier transform on $\mathbb{Z}$, we shall decompose the dual group into major and minor arcs. To this end suppose that $\eta>0$, and $a$ and $q$ are positive integers. We write $\left({ }^{2}\right)$

$$
\begin{aligned}
\mathfrak{M}_{a, q, \eta} & :=\{\theta \in \mathbb{T}:|\theta-a / q| \leq \eta\}, \\
\mathfrak{M}_{q, \eta}^{*} & :=\bigcup\left\{\mathfrak{M}_{a, q, \eta}: 1 \leq a \leq q \text { and }(a, q)=1\right\}, \\
\mathfrak{M}_{q, \eta} & :=\bigcup\left\{\mathfrak{M}_{a, q, \eta}: 1 \leq a \leq q\right\} .
\end{aligned}
$$

Often will there be a further parameter $Q$ with $q \leq Q$, in which case we will usually have $\eta=1 / q Q$ and write

$$
\mathfrak{M}_{a, q}:=\mathfrak{M}_{a, q,(q Q)^{-1}}, \quad \mathfrak{M}_{q}^{*}:=\mathfrak{M}_{q,(q Q)^{-1}}^{*}, \quad \mathfrak{M}_{q}:=\mathfrak{M}_{q,(q Q)^{-1}} .
$$

The quantity $Q$ will always be clear from the context.

4. A prime number theorem for arithmetic progressions. In this section we shall develop the small amount of number theory which we require. All the results we use are well known, although they are not always stated in the most useful fashion. We shall refer to the book [Dav00] of Davenport.

Suppose that $x$ is real and $a$ and $q$ are positive integers. Then we write

$$
\psi(x ; q, a):=\sum_{\substack{n \leq x \\ n \equiv a(\bmod q)}} \Lambda(n),
$$

where $\Lambda$ is the usual von Mangoldt function.

Estimating $\psi(x ; q, a)$ is one of the central problems in analytic number theory and to do so we introduce some auxiliary functions: For a Dirichlet character $\chi$ define

$$
\psi(x, \chi):=\sum_{n \leq x} \chi(n) \Lambda(n) .
$$

The analysis of $\psi(x, \chi)$ is, in turn, bound up in the analysis of the zeros of the corresponding $L$-function, $L(s, \chi)$, which is complicated by the possibility of a so-called exceptional zero; the following theorem limits the number of possible exceptions for a given Dirichlet character.

$\left({ }^{2}\right)$ Technically, elements of $\mathbb{T}$ are equivalence classes and so $|\cdot|$ is not well defined. We adopt the usual conventions in this regard. 
Theorem 4.1 ([Dav00, Chapter 14]). There is an absolute constant $c_{1}>0$ such that for any non-principal Dirichlet character $\chi$ of modulus $q$, $L(s, \chi)$ has at most one zero in the region

$$
\operatorname{Re} s \geq 1-\frac{c_{1}}{\log q(|\operatorname{Im} s|+3)} .
$$

This exceptional zero may only occur if $\chi$ is real, and then it is a simple real zero.

As usual, the analysis of the zeros of $L(s, \chi)$ can be reduced to the case when $\chi$ is primitive. Indeed, if $\chi$ has modulus $q$ and is induced by $\chi^{\prime}$ then, by the Euler product formula, we have

$$
L(s, \chi)=\prod_{p \mid q}\left(1-\chi^{\prime}(p) p^{-s}\right) L\left(s, \chi^{\prime}\right) \quad \text { for } \operatorname{Re} s>1 .
$$

Analytic continuation then tells us that in the region $\operatorname{Re} s>0$ we have $L(s, \chi)=0$ iff $L\left(s, \chi^{\prime}\right)=0$. Now, Landau showed that an exceptional zero can only occur for at most one primitive Dirichlet character:

Theorem 4.2 ([Dav00, Chapter 14]). There is an absolute constant $c_{2}>0$ such that for any distinct primitive real Dirichlet characters $\chi_{1}$ and $\chi_{2}$ with moduli $q_{1}$ and $q_{2}$, and real zeros $\beta_{1}$ and $\beta_{2}$ respectively, we have

$$
\min \left\{\beta_{1}, \beta_{2}\right\} \leq 1-\frac{c_{2}}{\log q_{1} q_{2}} .
$$

Write $c_{E}=\min \left\{c_{1}, c_{2}\right\}$ and suppose that $D \geq 2$ and $\chi$, a Dirichlet character, are given. We say that $\beta_{\chi}$ is an exceptional zero for $\chi$ at level $D$ if

$$
L\left(\beta_{\chi}, \chi\right)=0 \quad \text { and } \quad \operatorname{Re} \beta_{\chi} \geq 1-\frac{c_{E}}{\log 3 D} .
$$

The following corollary is an immediate consequence of Theorems 4.1 and 4.2.

Corollary 4.3. Suppose that $D \geq 2$. Then there is at most one primitive Dirichlet character $\chi_{D}$ and zero $\beta_{D}$ such that $\beta_{D}$ is an exceptional zero for $\chi_{D}$ at level $D$ and $\chi_{D}$ has modulus $q_{D} \leq D$.

If it exists, we call the Dirichlet character $\chi_{D}$ of the corollary the exceptional Dirichlet character at level $D$ and $\beta_{D}$ the exceptional zero at level $D$. In this event we shall need a bound on $\left(1-\beta_{D}\right)^{-1}$.

Proposition 4.4 ([Dav00, Chapter 14]). Suppose that $D \geq 2$ and the exceptional Dirichlet character at level $D$ exists and has zero $\beta_{D}$ and modulus $q_{D}$. Then $\left(1-\beta_{D}\right)^{-1}=O\left(q_{D}^{1 / 2} \log ^{2} q_{D}\right)$.

We require the following two prime number theorems.

TheOREM 4.5 ([Dav00, Chapter 20]). There is an absolute constant $c_{3}>0$ such that if $D \geq 2$ and $\chi$ is a non-principal Dirichlet character of modulus $q \leq D$, then: 
(i) if the exceptional Dirichlet character $\chi_{D}$ exists and $\chi$ is induced by $\chi_{D}$ then for any real $x \geq 1$ we have

$$
\psi(x, \chi)=-\frac{x^{\beta_{D}}}{\beta_{D}}+O\left(x \exp \left(-\frac{c_{3} \log x}{\sqrt{\log x}+\log D}\right)(\log D)^{2}\right)
$$

where $\beta_{D}$ is the exceptional zero;

(ii) if the exceptional Dirichlet character $\chi_{D}$ does not exist or $\chi$ is not induced by $\chi_{D}$ then for any real $x \geq 1$ we have

$$
\psi(x, \chi)=O\left(x \exp \left(-\frac{c_{3} \log x}{\sqrt{\log x}+\log D}\right)(\log D)^{2}\right) .
$$

TheOREM 4.6 ([Dav00, Chapter 20]). There is an absolute constant $c_{4}>0$ such that if $\chi^{\prime}$ is the principal Dirichlet character of modulus $q$, then for all real $x \geq 1$ we have

$$
\psi\left(x, \chi^{\prime}\right)=x+O\left(x \exp \left(-c_{4} \sqrt{\log x}\right)+\log q \log x\right) .
$$

Getting a handle on $\psi(x ; q, a)$ is now done via the identity

$$
\psi(x ; q, a)=\frac{1}{\phi(q)} \sum_{\chi} \bar{\chi}(a) \psi(x, \chi),
$$

where the summation is over all Dirichlet characters of modulus $q$. We can now prove the following proposition which is to be regarded as definitive for the terms $\left(D_{1}, D_{0}\right)$ is exceptional and $\left(D_{1}, D_{0}\right)$ is unexceptional.

Proposition 4.7. There is an absolute constant $c_{5}>0$ such that if $D_{1} \geq D_{0} \geq 2$, then at least one of the following two possibilities holds:

(i) $\left(\left(D_{1}, D_{0}\right)\right.$ is exceptional) There is a character $\chi_{D}$ of modulus $q_{D} \leq D_{0}$ and a real $\beta_{D}$ with $\left(1-\beta_{D}\right)^{-1}=O\left(q_{D}^{1 / 2} \log ^{2} q_{D}\right)$ such that for any real $x \geq 1$ and integers $a$ and $q$ with $1 \leq q q_{D} \leq D_{1}$ we have

$$
\begin{aligned}
\psi\left(x ; q q_{D}, a\right)= & \frac{\overline{\chi^{\prime}}(a) x}{\phi\left(q q_{D}\right)}-\frac{\overline{\chi^{\prime} \chi_{D}}(a) x^{\beta_{D}}}{\phi\left(q q_{D}\right) \beta_{D}} \\
& +O\left(x \exp \left(-\frac{c_{5} \log x}{\sqrt{\log x}+\log D_{1}}\right)\left(\log D_{1}\right)^{2}\right),
\end{aligned}
$$

where $\chi^{\prime}$ is the principal character of modulus $q q_{D}$.

(ii) $\left(\left(D_{1}, D_{0}\right)\right.$ is unexceptional) For any real $x \geq 1$ and integers $a$ and $q$ with $1 \leq q \leq D_{0}$ we have

$$
\psi(x ; q, a)=\frac{\overline{\chi^{\prime}}(a) x}{\phi(q)}+O\left(x \exp \left(-\frac{c_{5} \log x}{\sqrt{\log x}+\log D_{1}}\right)\left(\log D_{1}\right)^{2}\right),
$$

where $\chi^{\prime}$ is the principal Dirichlet character of modulus $q$.

Proof. Let $c_{5}:=\min \left\{c_{3}, c_{4}\right\}$. We split into two cases according to whether or not there is an exceptional character $\chi_{D}$ with modulus at most $D_{0}$. 
First, suppose that $\chi_{D}$ does exist, has zero $\beta_{D}$ and has modulus $q_{D} \leq D_{0}$. By Proposition $4.4,\left(1-\beta_{D}\right)^{-1}$ satisfies the appropriate bound. Now suppose that $x \geq 1$ is real and $a$ and $q$ are integers with $1 \leq q q_{D} \leq D_{1}$. Write $\chi^{\prime}$ for the principal character of modulus $q q_{D}$. There is exactly one character of modulus $q q_{D}$ induced by $\chi_{D}$ and that is $\chi^{\prime} \chi_{D}$. For this character, by Theorem 4.5(i), we have

$$
\psi\left(x, \chi^{\prime} \chi_{D}\right)=-\frac{x^{\beta_{D}}}{\beta_{D}}+O\left(x \exp \left(-\frac{c_{5} \log x}{\sqrt{\log x}+\log D_{1}}\right)\left(\log D_{1}\right)^{2}\right) .
$$

For all other non-principal characters $\chi$ we have, by Theorem 4.5(ii),

$$
\psi(x, \chi)=O\left(x \exp \left(-\frac{c_{5} \log x}{\sqrt{\log x}+\log D_{1}}\right)\left(\log D_{1}\right)^{2}\right) .
$$

Finally, by Theorem 4.6 we have

$$
\psi\left(x, \chi^{\prime}\right)=x+O\left(x \exp \left(-c_{5} \sqrt{\log x}\right)+\log D_{1} \log x\right) .
$$

Inserting these into (4.1) gives the first case of the proposition.

In the second case we suppose that either $\chi_{D}$ does not exist or, if it does, then it has modulus greater than $D_{0}$. Now suppose that $x \geq 1$ is real and $a$ and $q$ are integers with $1 \leq q \leq D_{0}$. Since $q$ is smaller than the modulus of $\chi_{D}$, if it exists at all, no character of modulus $q$ is induced by $\chi_{D}$, and we can apply Theorem 4.5(ii) to conclude that

$$
\psi(x, \chi)=O\left(x \exp \left(-\frac{c_{5} \log x}{\sqrt{\log x}+\log D_{1}}\right)\left(\log D_{1}\right)^{2}\right)
$$

for every non-principal $\chi$ of modulus $q$. Once again Theorem 4.6 gives

$$
\psi\left(x, \chi^{\prime}\right)=x+O\left(x \exp \left(-c_{5} \sqrt{\log x}\right)+\log D_{1} \log x\right),
$$

for $\chi^{\prime}$ the principal character of modulus $q$. Now inserting these estimates into (4.1) we find ourselves in the second case of the proposition.

5. The major arcs. We are interested in the Fourier transform of the von Mangoldt function $\Lambda$ and some closely related functions. Suppose that $N$ and $d$ are positive integers. We write

$$
\Lambda_{N, d}:= \begin{cases}\Lambda(d x+1) & \text { if } 1 \leq x \leq N \\ 0 & \text { otherwise. }\end{cases}
$$

We write $\Lambda_{N}$ as shorthand for $\Lambda_{N, 1}$. There will be two types of estimate for $\widehat{\Lambda}_{N, d}$ depending on whether or not a given pair of parameters $D_{1} \geq D_{0} \geq 2$ is exceptional or unexceptional. The reader may care to recall the definition from Proposition 4.7.

Before we begin, it will be useful to recall some standard definitions; the reader unfamiliar with this material may wish to consult the book [MV07]. 
For an integer $a$ and positive integer $q$ the Ramanujan sum $c_{q}(a)$ is defined by

$$
c_{q}(a):=\sum_{\substack{h=1 \\(h, q)=1}}^{q} e\left(\frac{h a}{q}\right)
$$

and moreover, $c_{q}(1)=\mu(q)$.

If positive integers $q$ and $d$ are coprime, write $m_{d, q}$ for a solution to $m_{d, q} d+1 \equiv 0(\bmod q)$. Then for any integers $a, q$ and $d$ with $q$ and $d$ positive we put

$$
\tau_{a, d, q}:=\sum_{\substack{m=0 \\(m d+1, q)=1}}^{q-1} e\left(m \frac{a}{q}\right)= \begin{cases}c_{q}(a) e\left(-m_{d, q} \frac{a}{q}\right) & \text { if }(d, q)=1 \\ 0 & \text { otherwise. }\end{cases}
$$

The proof of the equivalence of the sum with the expression in terms of the Ramanujan sum is a simple change of variables.

The remainder of this section provides major arc estimates for the two cases when the pair $\left(D_{1}, D_{0}\right)$ is exceptional and unexceptional.

5.1. Exceptional pairs. Throughout this subsection we assume that the pair $\left(D_{1}, D_{0}\right)$ is exceptional. We begin by estimating $\widehat{\Lambda}_{N, d}$ at a rational with small denominator and then extend the range.

LEMMA 5.2. There is an absolute constant $c_{6}>0$ such that for every set of non-negative integers $N, a, q, d$ with $d_{D} \mid d, 1 \leq d q \leq D_{1}$ and $N \geq 1$ we have

$\widehat{\Lambda}_{N, d}(a / q)=\frac{d N \tau_{a, d, q}}{\phi(d) \phi(q)}-\frac{(d N)^{\beta_{D}} \tau_{a, d, q}}{\phi(d) \phi(q) \beta_{D}}+O\left(N D_{1}^{2} \exp \left(-\frac{c_{6} \log N}{\sqrt{\log N}+\log D_{1}}\right)\right)$.

Proof. Note the formula

$$
\begin{aligned}
\widehat{\Lambda}_{N, d}(a / q) & =\sum_{\substack{x \leq d N+1 \\
x \equiv 1(\bmod d)}} \Lambda(x) e\left(\frac{a(x-1)}{d q}\right) \\
& =\sum_{m=0}^{q-1} e\left(m \frac{a}{q}\right) \sum_{\substack{x \leq d N+1 \\
x \equiv 1(\bmod d) \\
(x-1) / d \equiv m(\bmod q)}} \Lambda(x) \\
& =\sum_{m=0}^{q-1} e\left(m \frac{a}{q}\right) \psi(d N+1 ; d q, m d+1) .
\end{aligned}
$$

Since $\left(D_{1}, D_{0}\right)$ is exceptional we get a character $\chi_{D}$ of modulus $d_{D} \leq D_{0}$ and a real $\beta_{D}$ with $\left(1-\beta_{D}\right)^{-1}=O\left(d_{D}^{1 / 2} \log ^{2} d_{D}\right)$ such that for any real $x \geq 1$ 
and integers $a^{\prime}$ and $q^{\prime}$ with $1 \leq q^{\prime} d_{D} \leq D_{1}$ we have

$$
\begin{aligned}
\psi\left(x ; q^{\prime} d_{D}, a^{\prime}\right)= & \frac{\overline{\chi^{\prime}}\left(a^{\prime}\right) x}{\phi\left(q^{\prime} d_{D}\right)}-\frac{\overline{\chi^{\prime} \chi_{D}}\left(a^{\prime}\right) x^{\beta_{D}}}{\phi\left(q^{\prime} d_{D}\right) \beta_{D}} \\
& +O\left(x \exp \left(-\frac{c_{5} \log x}{\sqrt{\log x}+\log D_{1}}\right)\left(\log D_{1}\right)^{2}\right)
\end{aligned}
$$

where $\chi^{\prime}$ is the principal character of modulus $q^{\prime} d_{D}$. Now suppose that $d_{D} \mid d$ and $1 \leq d q \leq D_{1}$. There are three terms to consider when substituting (5.2), with $q^{\prime}=d q / d_{D}, x=d N+1$ and $a^{\prime}=m d+1$, into (5.1). First,

$$
\begin{aligned}
\sum_{m=0}^{q-1} e\left(m \frac{a}{q}\right) \frac{\overline{\chi^{\prime}}(m d+1)(d N+1)}{\phi(d q)} & =\frac{d N+1}{\phi(d q)} \sum_{\substack{m=0 \\
(m d+1, d q)=1}}^{q-1} e\left(m \frac{a}{q}\right) \\
& =\frac{(d N+1) \tau_{a, d, q}}{\phi(d) \phi(q)},
\end{aligned}
$$

recalling the definition of $\tau_{a, d, q}$ and the fact that it is zero unless $(d, q)=1$. Secondly, we have the sum

$$
\sum_{m=0}^{q-1} e\left(m \frac{a}{q}\right) \frac{\overline{\chi^{\prime} \chi_{D}}(m d+1)(d N+1)^{\beta_{D}}}{\phi(d q) \beta_{D}} .
$$

Since $\chi_{D}$ has modulus $d_{D}$ which divides $d$ we conclude that $\chi_{D}(m d+1)=$ $\chi_{D}(1)=1$ whatever the value of $m$, thus the above sum is equal to

$$
\sum_{m=0}^{q-1} e\left(m \frac{a}{q}\right) \frac{\overline{\chi^{\prime}}(m d+1)(d N+1)^{\beta_{D}}}{\phi(d q) \beta_{D}}=\frac{(d N+1)^{\beta_{D}} \tau_{a, d, q}}{\phi(d) \phi(q) \beta_{D}}
$$

by the same calculation as for the previous sum. Finally, we have an error term

$$
O\left(q(d N+1) \exp \left(-\frac{c_{5} \log (d N+1)}{\sqrt{\log (d N+1)}+\log D_{1}}\right)\left(\log D_{1}\right)^{2}\right),
$$

which is certainly

$$
O\left(N D_{1}^{2} \exp \left(-\frac{c_{6} \log N}{\sqrt{\log N}+\log D_{1}}\right)\right)
$$

for $c_{6}:=c_{5} / 4$. Combining these terms yields the lemma.

Proposition 5.3 (Major arcs estimate for exceptional pairs). For all non-negative integers $N, a, q, d$ with $d_{D} \mid d, 1 \leq d q \leq D_{1},(a, q)=1$ and $N \geq 1$, and elements $\theta \in \mathbb{T}$, we have

$$
\left|\widehat{\Lambda}_{N, d}(\theta)\right| \leq \frac{\left|\widehat{\Lambda}_{N, d}(0)\right|}{\phi(q)}+O\left((1+|\kappa| N) N D_{1}^{2} \exp \left(-\frac{c_{6} \log N}{\sqrt{\log N+\log D_{1}}}\right)\right),
$$


where $\kappa:=\theta-a / q$ and

$$
\left|\widehat{\Lambda}_{N, d}(0)\right| \gg \frac{N}{\phi(d)}+O\left(N D_{1}^{2} \exp \left(-\frac{c_{6} \log N}{\sqrt{\log N}+\log D_{1}}\right)\right) .
$$

Proof. Begin by applying Lemma 5.2 to deduce that for every set of non-negative integers $x, a, q, d$ with $d_{D} \mid d, 1 \leq d q \leq D$ and $1 \leq x \leq N$ we have

$$
\begin{aligned}
\widehat{\Lambda}_{x, d}(a / q)= & \frac{d x \tau_{a, d, q}}{\phi(d) \phi(q)}-\frac{(d x)^{\beta_{D}} \tau_{a, d, q}}{\phi(d) \phi(q) \beta_{D}} \\
& +O\left(N D_{1}^{2} \exp \left(-\frac{c_{6} \log N}{\sqrt{\log N}+\log D_{1}}\right)\right) .
\end{aligned}
$$

In particular we have

$$
\widehat{\Lambda}_{x, d}(0)=\frac{d x}{\phi(d)}-\frac{(d x)^{\beta_{D}}}{\phi(d) \beta_{D}}+O\left(N D_{1}^{2} \exp \left(-\frac{c_{6} \log N}{\sqrt{\log N}+\log D_{1}}\right)\right),
$$

and hence

$$
\widehat{\Lambda}_{x, d}(a / q)=\frac{\tau_{a, d, q}}{\phi(q)} \widehat{\Lambda}_{x, d}(0)+O\left(N D_{1}^{2} \exp \left(-\frac{c_{6} \log N}{\sqrt{\log N}+\log D_{1}}\right)\right) .
$$

Observe, by telescoping, that

$$
\widehat{\Lambda}_{N, d}(\theta)=\sum_{n=1}^{N}\left(\widehat{\Lambda}_{n, d}(a / q)-\widehat{\Lambda}_{n-1, d}(a / q)\right) e(\kappa n) .
$$

Integration by parts then tells us that

$$
\widehat{\Lambda}_{N, d}(\theta)=\left[\widehat{\Lambda}_{x, d}(a / q) e(\kappa x)\right]_{0}^{N}-2 \pi i \kappa \int_{0}^{N} \widehat{\Lambda}_{x, d}(a / q) e(\kappa x) d x .
$$

We use (5.5) to estimate the right hand side of this. The first term is

$$
\frac{\tau_{a, d, q} e(\kappa N)}{\phi(q)} \widehat{\Lambda}_{N, d}(0)+O\left(N D_{1}^{2} \exp \left(-\frac{c_{6} \log N}{\sqrt{\log N}+\log D_{1}}\right)\right) .
$$

We consider the second term on the right of (5.6) in two parts. First, note that

$$
\begin{array}{r}
2 \pi i \kappa \int_{0}^{N}\left(\widehat{\Lambda}_{x, d}(a / q)-\frac{d x \tau_{a, d, q}}{\phi(d) \phi(q)}+\frac{(d x)^{\beta_{D}} \tau_{a, d, q}}{\beta_{D} \phi(d) \phi(q)}\right) e(\kappa x) d x \\
=O\left(|\kappa| N^{2} D_{1}^{2} \exp \left(-\frac{c_{6} \log N}{\sqrt{\log N}+\log D_{1}}\right)\right)
\end{array}
$$

by (5.3). Secondly, note that

$$
2 \pi i \kappa \int_{0}^{N}\left(\frac{d x \tau_{a, d, q}}{\phi(d) \phi(q)}-\frac{(d x)^{\beta_{D}} \tau_{a, d, q}}{\beta_{D} \phi(d) \phi(q)}\right) e(\kappa x) d x
$$


is equal to

$$
\frac{\tau_{a, d, q}}{\phi(d) \phi(q)}\left[\left(d x-\frac{(d x)^{\beta_{D}}}{\beta_{D}}\right) e(\kappa x)\right]_{0}^{N}-\frac{\tau_{a, d, q}}{\phi(d) \phi(q)} \int_{0}^{N}\left(d-d^{\beta_{D}} x^{\beta_{D}-1}\right) e(\kappa x) d x,
$$

by integration by parts. The first term here is equal to

$$
\frac{\tau_{a, d, q} e(\kappa N)}{\phi(q)} \widehat{\Lambda}_{N, d}(0)+O\left(N D_{1}^{2} \exp \left(-\frac{c_{6} \log N}{\sqrt{\log N}+\log D_{1}}\right)\right),
$$

by (5.4). So, combining what we have so far we get that

$$
\begin{aligned}
\widehat{\Lambda}_{N, d}(\theta)= & \frac{\tau_{a, d, q}}{\phi(q) \phi(d)} \int_{0}^{N}\left(d-d^{\beta_{D}} x^{\beta_{D}-1}\right) e(\kappa x) d x \\
& +O\left((1+|\kappa| N) N D_{1}^{2} \exp \left(-\frac{c_{6} \log N}{\sqrt{\log N}+\log D_{1}}\right)\right) .
\end{aligned}
$$

Now, note that $d\left(1-(d x)^{\beta_{D}-1}\right) \geq 0$ if $d x \geq 1$, so

$$
\begin{aligned}
\left|\int_{0}^{N}\left(d-d^{\beta_{D}} x^{\beta_{D}-1}\right) e(\kappa x) d x\right| & \leq\left|\int_{1}^{N}\left(d-d^{\beta_{D}} x^{\beta_{D}-1}\right) e(\kappa x) d x\right|+O(1) \\
& \leq \int_{1}^{N}\left|d-d^{\beta_{D}} x^{\beta_{D}-1}\right| d x+O(1) \\
& =\int_{1}^{N}\left(d-d^{\beta_{D}} x^{\beta_{D}-1}\right) d x+O(1) \\
& =d N-\frac{(d N)^{\beta_{D}}}{\beta_{D}}+O(1) .
\end{aligned}
$$

Thus we conclude that the integral in (5.7) is bounded above in absolute value by

$$
\left|\widehat{\Lambda}_{N, d}(a / q)\right|+O\left((1+|\kappa| N) N D_{1}^{2} \exp \left(-\frac{c_{6} \log N}{\sqrt{\log N}+\log D_{1}}\right)\right) .
$$

Hence, by (5.5),

$$
\left|\widehat{\Lambda}_{N, d}(\theta)\right| \leq \frac{\left|\tau_{a, d, q}\right|}{\phi(q)}\left|\widehat{\Lambda}_{N, d}(0)\right|+O\left((1+|\kappa| N) N D_{1}^{2} \exp \left(-\frac{c_{6} \log N}{\sqrt{\log N}+\log D_{1}}\right)\right) .
$$

Now, if $(a, q)=1$ then $\left|\tau_{a, d, q}\right| \leq 1$ so we have the first part of the proposition.

To get the lower bound on $\left|\widehat{\Lambda}_{N, d}(0)\right|$ we return to (5.4). If $x \geq 16$ and $\varepsilon \in(0,1 / 2]$ then $1-x^{-\varepsilon} /(1-\varepsilon) \geq \varepsilon$, whence

$$
d N-\frac{(d N)^{\beta_{D}}}{\beta_{D}} \geq d N\left(\beta_{D}-1\right)+O(1) .
$$


Inserting the estimate for $\left(\beta_{D}-1\right)^{-1}$ (which we get since $\left(D_{1}, D_{0}\right)$ is exceptional) and recalling that $d \geq d_{D}$ yields the lower bound for $\left|\widehat{\Lambda}_{N, d}(0)\right|$.

5.4. Unexceptional pairs. In this subsection we assume that $\left(D_{1}, D_{0}\right)$ is unexceptional. The argument here is easier than that for exceptional pairs and proceeds as above except that terms involving the exceptional zero do not occur. We omit the details.

Proposition 5.5 (Major arcs estimate for unexceptional pairs). For every set of non-negative integers $N, a, q, d$ with $1 \leq d q \leq D_{0},(a, q)=1$ and $N \geq 1$, and elements $\theta \in \mathbb{T}$, we have

$$
\left|\widehat{\Lambda}_{N, d}(\theta)\right| \leq \frac{\left|\widehat{\Lambda}_{N, d}(0)\right|}{\phi(q)}+O\left((1+|\kappa| N) N D_{1}^{2} \exp \left(-\frac{c \log N}{\sqrt{\log N}+\log D_{1}}\right)\right),
$$

where $\kappa:=\theta-a / q$ and

$$
\left|\widehat{\Lambda}_{N, d}(0)\right| \geq \frac{d N}{\phi(d)}+O\left(N D_{1}^{2} \exp \left(-\frac{c \log N}{\sqrt{\log N}+\log D_{1}}\right)\right) .
$$

6. The minor arcs. The minor arcs are far easier to estimate than the major arcs were. We begin with Vinogradov's classical estimate, recalling that $\Lambda_{N}$ is shorthand for $\Lambda_{N, 1}$.

Theorem 6.1 ([Dav00, Chapter 25]). Suppose that $N$ and $q \leq Q$ are positive integers, $\theta \in \mathbb{T}$ and $a \in\{1, \ldots, q\}$ is coprime to $q$ and has $|\theta-a / q| \leq$ $1 / q Q$. Then

$$
\left|\widehat{\Lambda}_{N}(\theta)\right| \ll(\log N)^{4}\left(N / \sqrt{q}+N^{4 / 5}+\sqrt{N q}\right) .
$$

This has the following relevant corollary.

Corollary 6.2 (Minor arcs estimate). Suppose that $d \leq N$ and $q \leq Q$ are positive integers, $\theta \in \mathbb{T}$ and $a \in\{1, \ldots, q\}$ is coprime to $q$ and has $|\theta-a / q| \leq 1 / q Q$. Then

$$
\left|\widehat{\Lambda}_{N, d}(\theta)\right| \ll d(\log N)^{4}\left(N / \sqrt{q}+N^{4 / 5}+\sqrt{N Q}\right) .
$$

Proof. Begin by noting that

$$
\widehat{\Lambda}_{N, d}(\theta)=\frac{1}{d} \sum_{m=0}^{d-1} \sum_{x \leq d N+1} \Lambda(x) e\left(\theta \frac{x-1}{d}+\frac{m(x-1)}{d}\right),
$$

so

$$
\left|\widehat{\Lambda}_{N, d}(\theta)\right| \leq \frac{1}{d} \sum_{m=0}^{d-1}\left|\widehat{\Lambda}_{d N+1}((\theta+m) / d)\right| .
$$

Now, suppose that $m \in\{0, \ldots, d-1\}$ and write $\theta^{\prime}:=(\theta+m) / d$. We may apply Dirichlet's pigeon-hole principle to get a positive integer $q^{\prime} \leq$ 
$Q^{\prime}:=2 d Q$ and another $a^{\prime} \in\left\{1, \ldots, q^{\prime}\right\}$ with $\left(a^{\prime}, q^{\prime}\right)=1$ and such that $\left|\theta^{\prime}-a^{\prime} / q^{\prime}\right| \leq 1 / q^{\prime} Q^{\prime}$. So

$\left|a^{\prime} / q^{\prime}-(a+m q) / d q\right| \leq\left|\theta^{\prime}-a^{\prime} / q^{\prime}\right|+\left|\theta^{\prime}-(a+m q) / d q\right| \leq 1 / q^{\prime} Q^{\prime}+1 / d q Q$,

and hence

$$
\left|a^{\prime} d q-(a+m q) q^{\prime}\right| \leq 1 / 2+q^{\prime} / Q .
$$

The left hand side is an integer and if $q^{\prime}<q / 2$ then it is zero. This implies that $q \mid q^{\prime}$ since $(q, a+m q)=1$, whence $q^{\prime} \geq q$. This contradiction means that $q^{\prime} \geq q / 2$. Now we just apply Theorem 6.1 to the approximation $a^{\prime} / q^{\prime}$ (to $\theta^{\prime}$ ) to get the result.

7. Some energy increment lemmas. The main result of this section is an energy increment argument. Such arguments are common, and an example from a very similar context may be found in [Sze90] and [HB87].

We begin with a preliminary technical lemma.

Lemma 7.1. Suppose that $P$ is an arithmetic progression with common difference $d$ and $A \subset\{1, \ldots, N\}$ has $\alpha N$ elements. Suppose further that

$$
\sum_{x \in \mathbb{Z}}\left(1_{A}-\alpha 1_{[N]}\right) * 1_{P}(x)^{2} \geq c \alpha^{2} N|P|^{2} .
$$

Then there is an integer $x^{\prime} \in \mathbb{Z}$ such that

$$
1_{A} * 1_{P}\left(x^{\prime}\right) \geq \alpha(1+c)|P|+O\left(N^{-1} d|P|^{2}\right) .
$$

Proof. First note that

$$
\begin{aligned}
\alpha \sum_{x \in \mathbb{Z}} 1_{A} * 1_{P}(x) 1_{[N]} * 1_{P}(x) & =\alpha \sum_{x \in \mathbb{Z}} 1_{A}(x) 1_{[N]} * 1_{P} * 1_{P}(x) \\
& =\alpha^{2} N|P|^{2}+O\left(\alpha d|P|^{3}\right)
\end{aligned}
$$

and

$$
\alpha^{2} \sum_{x \in \mathbb{Z}} 1_{[N]} * 1_{P}(x)^{2}=\alpha^{2} N|P|^{2}+O\left(\alpha^{2} d|P|^{3}\right) .
$$

Expanding the hypothesis we deduce that

$$
\sum_{x \in \mathbb{Z}} 1_{A} * 1_{P}(x)^{2} \geq(1+c) \alpha^{2} N|P|^{2}+O\left(\alpha d|P|^{3}\right) .
$$

Now Hölder's inequality yields

$$
\sup _{x \in \mathbb{Z}} 1_{A} * 1_{P}(x) \alpha N|P| \geq \sum_{x \in \mathbb{Z}} 1_{A} * 1_{P}(x)^{2},
$$

from which the result follows.

The next result is a fairly common form of the energy increment argument. It may be useful to recall the definition of the intervals $\mathfrak{M}_{a, q, \eta}$ from $\S 3$ before reading further. 
Proposition 7.2. Suppose that $\eta>0, N$ and $q$ are positive integers, and $A \subset\{1, \ldots, N\}$ has $\alpha N$ elements. Write

$$
E_{A, q, \eta}:=\alpha^{-1}|A|^{-1} \int_{\theta \in \mathfrak{M}_{q, \eta}}\left|\left(1_{A}-\alpha 1_{[N]}\right)^{\wedge}(\theta)\right|^{2} d \theta .
$$

Then there is an arithmetic progression $P$ with common difference $q$ and $|P| \gg q^{-1} \min \left\{\eta^{-1}, E_{A, q, \eta}|A|\right\}$ such that $|A \cap P| \geq \alpha\left(1+E_{A, q, \eta} / 4\right)|P|$.

Proof. Let $P$ be the progression of common difference $q$ and length $2 M+1$ centred at the origin; we shall optimize for $M$ later. In this case (by scaling the Dirichlet kernel) we have

$$
\widehat{1}_{P}(\theta)=\frac{\sin (|P| \pi q \theta)}{\sin (\pi q \theta)}
$$

with the usual convention at $\theta=0$. Now suppose that $\theta \in \mathfrak{M}_{q, \eta}$, so that there is some integer $a$ with $|\theta-a / q| \leq \eta$. Thus, writing $\kappa:=\theta-a / q$ and recalling the inequalities $|\sin x| \geq 2|x| / \pi$ if $|x| \leq \pi / 2$ and $|\sin x| \leq|x|$, we have

$$
\left|\widehat{1}_{P}(\theta)\right|=\left|\frac{\sin (|P| \pi q \kappa)}{\sin (\pi q \kappa)}\right| \geq \frac{2|| P|q \kappa|}{|\pi q \kappa|}=\frac{2|P|}{\pi}
$$

provided $|P| q \eta \leq 1 / 2$. It follows that

$$
\int_{\theta \in \mathfrak{M}_{q, \eta}}\left|\left(1_{A}-\alpha 1_{[N]}\right)^{\wedge}(\theta)\right|^{2}\left|\widehat{1}_{P}(\theta)\right|^{2} d \theta \geq \frac{4}{\pi^{2}} E_{A, q, \eta} \alpha|A||P|^{2} .
$$

Now the left hand side is certainly dominated by the same integral without the restriction of the domain of integration and hence, by Parseval's theorem applied to the unrestricted domain, we have

$$
\sum_{x \in \mathbb{Z}}\left(1_{A}-\alpha 1_{[N]}\right) * 1_{P}(x)^{2} \geq \frac{4}{\pi^{2}} E_{A, q}|A|^{2}|P|^{2} .
$$

Now we may apply Lemma 7.1 to get some $x^{\prime} \in \mathbb{Z}$ such that

$$
1_{A} * 1_{P}\left(x^{\prime}\right) \geq\left(1+4 E_{A, q, \eta} / \pi^{2}\right) \alpha|P|+O\left(N^{-1} q|P|^{2}\right) .
$$

It follows that there is a choice of $M \gg q^{-1} \min \left\{\eta^{-1}, E_{A, q}|A|\right\}$ for which

$$
1_{A} * 1_{P}\left(x^{\prime}\right) \geq\left(1+E_{A, q, \eta} / 4\right) \alpha|P| .
$$

For our work we shall use the following corollary which is designed specifically for the problem we are considering.

Corollary 7.3. Suppose that $N$ is a positive integer, $A \subset\{1, \ldots, N\}$ has $\alpha N$ elements, $Q^{\prime} \geq 1$ and $Q:=N / Q^{\prime}$. For each $q$ with $1 \leq q \leq Q^{\prime}$ write

$$
E_{A, q}^{*}:=\alpha^{-1}|A|^{-1} \int_{\theta \in \mathfrak{M}_{q}^{*}}\left|\left(1_{A}-\alpha 1_{[N]}\right)^{\wedge}(\theta)\right|^{2} d \theta,
$$


and suppose that

$$
\sum_{q=1}^{Q^{\prime}} \frac{1}{\phi(q)} E_{A, q}^{*} \geq c
$$

Then there is an arithmetic progression $P$ with common difference $q \leq Q^{\prime}$ and $|P| \gg Q^{\prime-1} N \min \left\{Q^{\prime-1}, \alpha c\right\}$ such that $|A \cap P| \geq \alpha\left(1+2^{-5} c\right)|P|$.

Proof. For $\eta>0$ we define $E_{A, q, \eta}$ as in Proposition 7.2 and write

$$
I_{A, a, q, \eta}:=\alpha^{-1}|A|^{-1} \int_{\theta \in \mathfrak{M}_{a, q, \eta}}\left|\left(1_{A}-\alpha 1_{[N]}\right)^{\wedge}(\theta)\right|^{2} d \theta .
$$

Begin by noting that

$$
\begin{aligned}
\sum_{q=1}^{Q^{\prime}} \frac{q}{\phi(q)} E_{A, q, Q^{-1}} & =\sum_{q=1}^{Q^{\prime}} \frac{q}{\phi(q)} \sum_{r=1}^{q} I_{A, r, q, Q^{-1}} \\
& =\sum_{q=1}^{Q^{\prime}} \frac{q}{\phi(q)} \sum_{q^{\prime} h=q} \sum_{\substack{r^{\prime}=1 \\
\left(r, q^{\prime}\right)=1}}^{q^{\prime}} I_{A, r^{\prime} h, q^{\prime} h, Q^{-1}}
\end{aligned}
$$

but this last expression is equal to

$$
\sum_{q=1}^{Q^{\prime}} \frac{q}{\phi(q)} \sum_{q^{\prime} h=q} E_{A, q^{\prime}, Q^{-1}}^{*}=\sum_{q^{\prime}=1}^{Q^{\prime}} E_{A, q^{\prime}, Q^{-1}}^{*} \sum_{h=1}^{Q^{\prime} / q^{\prime}} \frac{q^{\prime} h}{\phi\left(q^{\prime} h\right)}
$$

Now we also have

$$
\sum_{h=1}^{Q^{\prime} / q^{\prime}} \frac{q^{\prime} h}{\phi\left(q^{\prime} h\right)} \geq \frac{q^{\prime}}{\phi\left(q^{\prime}\right)} \sum_{h=1}^{Q^{\prime} / q^{\prime}} 1 \geq \frac{Q^{\prime}}{2 \phi\left(q^{\prime}\right)}
$$

and so

$$
\sum_{q=1}^{Q^{\prime}} \frac{q}{\phi(q)} E_{A, q, Q^{-1}} \geq \frac{Q^{\prime}}{2} \sum_{q^{\prime}=1}^{Q^{\prime}} \frac{1}{\phi\left(q^{\prime}\right)} E_{A, q^{\prime}, Q^{-1}}^{*} \geq \frac{Q^{\prime} c}{2}
$$

by hypothesis and the fact that $1 / q Q \leq 1 / Q$. But it is well known (see, for example, the book [MV07]) that

$$
\sum_{q=1}^{Q^{\prime}} \frac{q}{\phi(q)} \leq 4 Q^{\prime}
$$

so, by a trivial instance of Hölder's inequality, we conclude that there is some $q$ with $1 \leq q \leq Q^{\prime}$ such that $E_{A, q, Q^{-1}} \geq c / 8$. We now apply Proposition 7.2 to get the result. 
8. The main iteration lemma. Our main argument is basically iterative - although the eventual proof will be by a maximality argument - and the central lemma follows. Essentially it says that if none of the various input parameters is too small and $A-A$, that is, the set of differences between elements of $A$, misses all numbers of the form $(p-1) / d$, then $A$ must have much larger density on a reasonable sub-progression.

Lemma 8.1 (Iteration lemma). Suppose that $D_{1} \geq D_{0} \geq 2, A \subset\{1, \ldots, N\}$ has $\alpha N$ elements and either

(1) $\left(D_{1}, D_{0}\right)$ is exceptional and $d \leq D_{1}$ is such that $d_{D} \mid d$; or

(2) $\left(D_{1}, D_{0}\right)$ is unexceptional and $d \leq D_{0}$.

Then there are absolute constants $c_{8}, c_{9}, c_{10}>0$ such that at least one of the following holds:

(i) (Density increment) There is an integer $d^{\prime}$ such that $d^{\prime}=O\left(\alpha^{-2}\right)$ and a progression $P$ of common difference $d^{\prime}$ and length at least $\left(c_{9} \alpha / d \log N\right)^{8} N$ such that $|A \cap P| \geq \alpha\left(1+c_{8}\right)|P|$.

(ii) (Structure in difference set) $A-A$ contains a number of the form $(p-1) / d$ with $p$ a prime.

(iii) ( $N$ is small) $N \leq O\left(\exp \left(c_{10} \log ^{2} D_{1}\right)\right)$.

(iv) ( $d$ is large or $\alpha$ is small) Either

(a) $\left(D_{1}, D_{0}\right)$ is exceptional, and $d^{-1}=O\left(D_{1}^{-c_{10}}\right)$ or $\alpha=O\left(D_{1}^{-c_{10}}\right)$; or

(b) $\left(D_{1}, D_{0}\right)$ is unexceptional, and $d^{-1}=O\left(D_{0}^{-c_{10}} / \log ^{2} D_{1}\right)$ or $\alpha=$ $O\left(D_{0}^{-c_{10}} / \log ^{2} D_{1}\right)$.

(v) ( $\alpha$ is small) $\alpha=O\left(D_{1}^{-c_{10}}\right)$.

Proof. Throughout the proof we shall introduce constants $c, c^{\prime}, c^{\prime \prime}, \ldots$ which will each be optimized at some later point and will end up being absolute positive constants. The reason for this slightly unappealing approach is that we have not been explicit about any of the constants in the error terms we have so far produced.

Let $c>0$ be some constant to be optimized later. Either $N \leq c \alpha^{-1}$ (and we shall, once we have shown we can choose $c$ to be absolute, be in outcome (iii) or (v)), or the integer $N^{\prime}=\left\lfloor c^{\prime} \alpha N\right\rfloor$ has $N^{\prime} \geq 1$.

Irrespective of whether $\left(D_{1}, D_{0}\right)$ is exceptional or unexceptional we see, from Propositions 5.3 or 5.5, that

$$
\left|\widehat{\Lambda}_{N^{\prime}, d}(0)\right| \gg \frac{N^{\prime}}{\phi(d)}+O\left(N^{\prime} D_{1}^{2} \exp \left(-\frac{c_{6} \log N^{\prime}}{\sqrt{\log N^{\prime}}+\log D_{1}}\right)\right) .
$$

Now $\phi(d) \leq d \leq D_{1}$, so either $N \leq c^{-1} \alpha^{-1} \exp \left(O\left(\log ^{2} D_{1}\right)\right)$ (and we shall 
be in outcome (iii) or (v) again) or we have the estimate

$$
\left|\widehat{\Lambda}_{N^{\prime}, d}(0)\right| \gg \frac{N^{\prime}}{\phi(d)} .
$$

Write $I$ for the interval $[N]$ and consider the inner product

$$
\left\langle\left(1_{A}-\alpha 1_{I}\right) *\left(1_{-A}-\alpha 1_{-I}\right), \Lambda_{N^{\prime}, d}\right\rangle .
$$

If $A-A$ contains a number of the form $(p-1) / d$ for some prime $p$ then we are in outcome (ii) of the lemma; consequently, assume that it does not. In this case the only integers $x$ which support a contribution in the inner product $\left\langle 1_{A} * 1_{-A}, \Lambda_{N^{\prime}, d}\right\rangle$ are those for which $d x+1$ is a prime power with the power strictly greater than one. There are at most $O\left(\sqrt{d N^{\prime}}\right)$ such integers and furthermore $\left\|\Lambda_{N^{\prime}, d}\right\|_{\ell^{\infty}(\mathbb{Z})}=O\left(\log d N^{\prime}\right)$ and $\left\|1_{A} * 1_{-A}\right\|_{\ell^{\infty}(\mathbb{Z})} \leq \alpha N$, whence

$$
\left\langle 1_{A} * 1_{-A}, \Lambda_{N^{\prime}, d}\right\rangle=O\left(\alpha N \sqrt{d N^{\prime}} \log d N^{\prime}\right) .
$$

We conclude that

$$
\left\langle 1_{A} * 1_{-A}, \Lambda_{N^{\prime}, d}\right\rangle=O\left(\alpha\left|\widehat{\Lambda}_{N^{\prime}, d}(0)\right| N^{\prime}\right)
$$

unless $N \leq \exp \left(O\left(\log \alpha^{-1} D_{1} c^{-1}\right)\right)$ (in which case we shall be in outcome (iii) or (v) again).

The other terms arising from expanding out (8.1) are more easily handled:

$$
\begin{aligned}
\left\langle 1_{I} * 1_{-A}, \Lambda_{N^{\prime}, d}\right\rangle & =\widehat{\Lambda}_{N^{\prime}, d}(0) \alpha N+O\left(\left|\widehat{\Lambda}_{N^{\prime}, d}(0)\right| N^{\prime}\right), \\
\left\langle 1_{A} * 1_{-I}, \Lambda_{N^{\prime}, d}\right\rangle & =\widehat{\Lambda}_{N^{\prime}, d}(0) \alpha N+O\left(\left|\widehat{\Lambda}_{N^{\prime}, d}(0)\right| N^{\prime}\right), \\
\left\langle 1_{I} * 1_{-I}, \Lambda_{N^{\prime}, d}\right\rangle & =\widehat{\Lambda}_{N^{\prime}, d}(0) N+O\left(\left|\widehat{\Lambda}_{N^{\prime}, d}(0)\right| N^{\prime}\right) .
\end{aligned}
$$

Thus it follows that

$$
\left\langle\left(1_{A}-\alpha 1_{I}\right) *\left(1_{-A}-\alpha 1_{-I}\right), \Lambda_{N^{\prime}, d}\right\rangle=\alpha^{2} N \widehat{\Lambda}_{N^{\prime}, d}(0)(-1+O(c)) .
$$

Now we pick $c \gg 1$ such that

$$
\left|\left\langle\left(1_{A}-\alpha 1_{I}\right) *\left(1_{-A}-\alpha 1_{-I}\right), \Lambda_{N^{\prime}, d}\right\rangle\right| \gg \alpha^{2} N\left|\widehat{\Lambda}_{N^{\prime}, d}(0)\right|,
$$

and apply Plancherel's theorem to the left hand side to get a Fourier space expression

$$
\int\left|\left(1_{A}-\alpha 1_{I}\right)^{\wedge}(\theta)\right|^{2}\left|\widehat{\Lambda}_{N^{\prime}, d}(\theta)\right| d \theta \gg \alpha^{2} N\left|\widehat{\Lambda}_{N^{\prime}, d}(0)\right| .
$$

Let $c^{\prime}>0$ be another constant to be optimized later. Write

$$
Q^{\prime}:=\frac{d^{4} \log ^{8} N}{c^{\prime 2} \alpha^{2}}, \quad Q:=N^{\prime} / Q^{\prime}, \quad \mathfrak{M}^{\prime}:=\bigcup_{q<Q^{\prime}} \mathfrak{M}_{q}^{*}, \quad \mathfrak{M}:=\bigcup_{Q^{\prime} \leq q \leq Q} \mathfrak{M}_{q}^{*}
$$

By Dirichlet's pigeon-hole principle $\mathbb{T}=\mathfrak{M} \cup \mathfrak{M}^{\prime}$ and so by the triangle 
inequality we have

$$
\begin{aligned}
\int\left|\left(1_{A}-\alpha 1_{I}\right)^{\wedge}(\theta)\right|^{2}\left|\widehat{\Lambda}_{N^{\prime}, d}(\theta)\right| d \theta \leq & \int_{\theta \in \mathfrak{M}^{\prime}}\left|\left(1_{A}-\alpha 1_{I}\right)^{\wedge}(\theta)\right|^{2}\left|\widehat{\Lambda}_{N^{\prime}, d}(\theta)\right| d \theta \\
& +\int_{\theta \in \mathfrak{M}}\left|\left(1_{A}-\alpha 1_{I}\right)^{\wedge}(\theta)\right|^{2}\left|\widehat{\Lambda}_{N^{\prime}, d}(\theta)\right| d \theta .
\end{aligned}
$$

Corollary 6.2 tells us that either $N^{\prime} \leq d$ (and we are in outcome (iii) or (v), since $d \leq D_{1}$ ), or else

$$
\left|\widehat{\Lambda}_{N^{\prime}, d}(\theta)\right| \ll d \log ^{4} N^{\prime}\left(N^{\prime} / \sqrt{q}+N^{4 / 5}+\sqrt{N^{\prime} Q}\right)
$$

for $\theta \in \mathfrak{M}_{q}^{*}$ and $q \leq Q$. So, if $\theta \in \mathfrak{M}$ then

$$
\left|\widehat{\Lambda}_{N^{\prime}, d}(\theta)\right| \ll \frac{N^{\prime}}{d}\left(c^{\prime} \alpha+d^{2} N^{\prime-1 / 10}\right) .
$$

Once again, either $N^{\prime} \leq c^{-10} d^{20} \alpha^{-10}$ (and we are in outcome (iii) or (v), since $d \leq D_{1}$ ), or we have

$$
\left|\widehat{\Lambda}_{N^{\prime}, d}(\theta)\right| \ll c^{\prime} \alpha\left|\widehat{\Lambda}_{N^{\prime}, d}(0)\right| \quad \text { for all } \theta \in \mathfrak{M} .
$$

It follows that

$$
\begin{aligned}
\int_{\theta \in \mathfrak{M}}\left|\left(1_{A}-\alpha 1_{I}\right)^{\wedge}(\theta)\right|^{2}\left|\widehat{\Lambda}_{N^{\prime}, d}(\theta)\right| d \theta & \ll c^{\prime} \alpha\left|\widehat{\Lambda}_{N^{\prime}, d}(0)\right| \int\left|\left(1_{A}-\alpha 1_{I}\right)^{\wedge}(\theta)\right|^{2} d \theta \\
& \ll c^{\prime} \alpha^{2} N\left|\widehat{\Lambda}_{N^{\prime}, d}(0)\right|,
\end{aligned}
$$

by Parseval's theorem. Hence we can choose $c^{\prime} \gg 1$ so that

$$
\int_{\theta \in \mathfrak{M}^{\prime}}\left|\left(1_{A}-\alpha 1_{I}\right)^{\wedge}(\theta)\right|^{2}\left|\widehat{\Lambda}_{N^{\prime}, d}(\theta)\right| d \theta \gg \alpha^{2} N\left|\widehat{\Lambda}_{N^{\prime}, d}(0)\right| .
$$

If $\left(D_{1}, D_{0}\right)$ is unexceptional and $d Q^{\prime}>D_{0}$ then we are in outcome (iii) or (iv), or else we can apply Proposition 5.5; if $\left(D_{1}, D_{0}\right)$ is exceptional and $d Q^{\prime}>D_{1}$ then we are in outcome (iii) or (iv), or else we can apply Proposition 5.3. So either we are done or we could apply the appropriate proposition and get

$$
\left|\widehat{\Lambda}_{N^{\prime}, d}(\theta)\right| \leq \frac{\left|\widehat{\Lambda}_{N^{\prime}, d}(0)\right|}{\phi(q)}+O\left(\left(1+Q^{-1} N^{\prime}\right) N^{\prime} D_{1}^{2} \exp \left(-\frac{c_{6} \log N^{\prime}}{\sqrt{\log N^{\prime}}+\log D_{1}}\right)\right)
$$

In view of the definition of $Q$ this error term is

$$
O\left(\alpha^{-2}\left(\log ^{8} N\right) N^{\prime} D_{1}^{6} \exp \left(-\frac{c_{6} \log N^{\prime}}{\sqrt{\log N^{\prime}}+\log D_{1}}\right)\right)
$$

and so once again either we are in outcome (iii) or (v) of the lemma, or

$$
\sup _{\theta \in \mathfrak{M}_{q}^{*}}\left|\widehat{\Lambda}_{N^{\prime}, d}(\theta)\right| \ll \frac{\left|\widehat{\Lambda}_{N^{\prime}, d}(0)\right|}{\phi(q)} \quad \text { for all } q \leq Q^{\prime}
$$


Set $Q^{\prime \prime}:=c^{\prime \prime-2} \alpha^{-2}$ for some $c^{\prime \prime}>0$ which will be chosen shortly. Write

$$
\mathfrak{M}^{\prime \prime}:=\bigcup_{Q^{\prime \prime} \leq q<Q^{\prime}} \mathfrak{M}_{q}^{*}, \quad \mathfrak{M}^{\prime \prime \prime}:=\bigcup_{q<Q^{\prime \prime}} \mathfrak{M}_{q}^{*},
$$

so that $\mathfrak{M}^{\prime}=\mathfrak{M}^{\prime \prime} \cup \mathfrak{M}^{\prime \prime \prime}$. Then

$$
\int_{\theta \in \mathfrak{M}^{\prime \prime}}\left|\left(1_{A}-\alpha 1_{I}\right)^{\wedge}(\theta)\right|^{2}\left|\widehat{\Lambda}_{N^{\prime}, d}(\theta)\right| d \theta \ll c^{\prime \prime} \alpha^{2} N\left|\widehat{\Lambda}_{N^{\prime}, d}(0)\right|
$$

by Parseval's theorem and (8.2) since $\phi(n) \gg n^{1 / 2}$; pick $c^{\prime \prime} \gg 1$ so that

$$
\int_{\theta \in \mathfrak{M}^{\prime \prime \prime}}\left|\left(1_{A}-\alpha 1_{I}\right)^{\wedge}(\theta)\right|^{2}\left|\widehat{\Lambda}_{N^{\prime}, d}(\theta)\right| d \theta \gg \alpha^{2} N\left|\widehat{\Lambda}_{N^{\prime}, d}(0)\right| .
$$

Now, by the triangle inequality we get

$$
\sum_{q=1}^{Q^{\prime \prime}} \int_{\theta \in \mathfrak{M}_{q}^{*}}\left|\left(1_{A}-\alpha 1_{I}\right)^{\wedge}(\theta)\right|^{2}\left|\widehat{\Lambda}_{N^{\prime}, d}(\theta)\right| d \theta \gg \alpha^{2} N\left|\widehat{\Lambda}_{N^{\prime}, d}(0)\right|,
$$

and so by (8.2),

$$
\sum_{q=1}^{Q^{\prime \prime}} \frac{\left|\widehat{\Lambda}_{N^{\prime}, d}(0)\right|}{\phi(q)} \int_{\theta \in \mathfrak{M}_{q}^{*}}\left|\left(1_{A}-\alpha 1_{I}\right)^{\wedge}(\theta)\right|^{2} d \theta \gg \alpha^{2} N\left|\widehat{\Lambda}_{N^{\prime}, d}(0)\right| .
$$

Thus by Corollary 7.3 (with the fact that $\left|\widehat{\Lambda}_{N^{\prime}, d}(0)\right|>0$ ) we find ourselves in the first case of the lemma.

9. Proof of Theorem 1.1. The main argument is now fairly straightforward. We begin with a preliminary technical lemma.

Lemma 9.1. Suppose that $A \subset\{1, \ldots, N\}$ has $\alpha N$ elements and $d$ is an integer. Then there is a progression $P$ with common difference $d$ and $|P| \gg \alpha N / d$ such that $|A \cap P| \geq \alpha|P| / 2$.

Proof. Let $P^{\prime}$ be a progression with common difference $d$. Then

$$
\sum_{x \in \mathbb{Z}}\left|1_{[N]} * 1_{P^{\prime}}(x)-\right| P^{\prime}\left|1_{[N]}(x)\right|=O\left(d\left|P^{\prime}\right|^{2}\right)
$$

whence

$$
|A|\left|P^{\prime}\right|=\left\langle 1_{A}, 1_{[N]} * 1_{P^{\prime}}\right\rangle+O\left(d\left|P^{\prime}\right|^{2}\right)=\left\langle 1_{A} * 1_{P^{\prime}}, 1_{[N]}\right\rangle+O\left(d\left|P^{\prime}\right|^{2}\right) .
$$

It follows that we can pick $\left|P^{\prime}\right| \gg \alpha N / d$ such that

$$
\left\langle 1_{A} * 1_{P^{\prime}}, 1_{[N]}\right\rangle \geq \alpha N\left|P^{\prime}\right| / 2 .
$$

Thus, by Hölder's inequality, there is some translate $P$ of $P^{\prime}$ with the desired property.

We now turn to the main proof. 
Proof of Theorem 1.1. Write $I$ for the interval $[N]$. We fix $D_{1} \geq D_{0} \geq 2$, to be optimized at the end of the argument, and put

$$
D_{1}=D_{0}^{\max \left\{2 c_{10}^{-1}, 1\right\}},
$$

where $c_{10}$ is the absolute constant from Lemma 8.1. We consider two cases according to whether $\left(D_{1}, D_{0}\right)$ is exceptional or unexceptional.

CASE 1: $\left(D_{1}, D_{0}\right)$ is exceptional. This gives us an integer $d_{D} \leq D_{0}$ with a number of properties. By Lemma 9.1 there is a progression $P$ with common difference $d_{D}$ and $|P| \gg \alpha N / d_{D}$ such that $|A \cap P| \geq \alpha|P| / 2$. Let $I_{D}:=$ $\{1, \ldots,|P|\}$ and let $A_{D}$ be the affine transformation of $A \cap P$ so that it lies in $I_{D}$. We write $\alpha_{D}$ for the density of $A$ in $I_{D}$ and $N_{D}$ for the length of $I_{D}$. Thus

$$
\alpha_{D} \geq \alpha / 2 \text { and } N_{D} \gg \alpha N / D_{0} .
$$

Furthermore, by the hypothesis on $A, A_{D}-A_{D}$ does not contain any numbers of the form $(p-1) / d_{D}$ with $p$ a prime. Let $\eta>0$ be a parameter to be optimized later and let $P^{\prime}$ be an arithmetic progression such that $\alpha^{\prime} N^{\prime \eta^{2}} d^{\prime-\eta}$ is maximal, where $\alpha^{\prime}$ is the relative density of $A_{D}$ on $P^{\prime}$, that is, $\left|A_{D} \cap P^{\prime}\right| /\left|P^{\prime}\right|$, and where $N^{\prime}$ is the length of $P^{\prime}$ and $d^{\prime}$ is the common difference of $P^{\prime}$. The choice of $\eta^{2}$ and $\eta$ is made with the benefit of hindsight; we could use two different parameters and optimize for both at the end.

In view of the maximality of $P^{\prime}$, we have $\alpha_{D} N_{D}^{\eta^{2}} \leq \alpha^{\prime} N^{\prime \eta^{2}} d^{\prime-\eta}$. Now, since $\alpha^{\prime} \leq 1, d^{\prime} \geq 1$ and $N^{\prime} \leq N_{D}$ it follows that

$$
\alpha_{D} \leq \alpha^{\prime}, d^{\prime} \leq \alpha_{D}^{-\eta^{-1}} \text { and } \quad N^{\prime} \geq \alpha_{D}^{\eta^{-2}} N_{D}
$$

whence

$$
\alpha \ll \alpha^{\prime}, \quad \log d^{\prime} \ll \eta^{-1} \log \alpha^{-1}
$$

and

$$
\log N=\log N^{\prime}+O\left(\eta^{-2} \log \alpha^{-1}+\log D_{0}\right) .
$$

Again, let $I^{\prime}:=\left\{1, \ldots, N^{\prime}\right\}$ and $A^{\prime}$ be the affine transformation of $A_{D} \cap P^{\prime}$ so that it lies in $I^{\prime}$. Apply Lemma 8.1 to get the following possibilities: either

(i) there is a progression $P^{\prime \prime}$ of common difference $d^{\prime \prime}=O\left(\alpha^{\prime-2}\right)$ and length at least $\left(c_{9} \alpha^{\prime} / d^{\prime} d_{D} \log N^{\prime}\right)^{8} N^{\prime}$ with $\left|A^{\prime} \cap P^{\prime \prime}\right| \geq \alpha^{\prime}\left(1+c_{8}\right)\left|P^{\prime \prime}\right|$; or

(ii) $A^{\prime}-A^{\prime}$ contains a number of the form $(p-1) / d^{\prime} d_{D}$ with $p$ a prime; or

(iii) $N^{\prime} \leq O\left(\exp \left(c_{10} \log ^{2} D_{1}\right)\right)$ whence, by $(9.2), \log N \ll \eta^{-2} \log \alpha^{-1}+$ $\log ^{2} D_{1}$; or

(iv) $\left(d^{\prime} d_{D}\right)^{-1}=O\left(D_{1}^{-c_{10}}\right)$ whence, by the relationship between $D_{0}$ and $D_{1}$, we get $d^{\prime} \gg D_{1}^{-c_{10} / 2}$ and so, by (9.1), $\log D_{1} \ll \eta^{-1} \log \alpha^{-1}$; or

(v) $\alpha^{\prime}=O\left(D_{1}^{-c_{10}}\right)$. 
In the first case, the maximal way in which $P^{\prime}$ was chosen ensures that

$$
\alpha^{\prime} N^{\prime \eta^{2}} d^{\prime-\eta} \geq \alpha^{\prime}\left(1+c_{8}\right)\left(N^{\prime}\left(c_{9} \alpha^{\prime} / d^{\prime} d_{D} \log N^{\prime}\right)^{8}\right)^{\eta^{2}}\left(c \alpha^{\prime}\right)^{-2 \eta} d^{\prime-\eta},
$$

from which we conclude

$$
\eta^{-2} \ll \eta^{-1} \log \alpha^{\prime-1}+\log D_{0}+\log d^{\prime}+\log \log N^{\prime} .
$$

Inserting the bounds from (9.1) and (9.2) and the fact that $\log D_{0} \ll \log D_{1}$ we get

$$
\eta^{-2} \ll \eta^{-1} \log \alpha^{-1}+\log D_{1}+\log \log N,
$$

and hence, by solving the quadratic,

$$
\eta^{-1} \ll \log \alpha^{-1}+\sqrt{\log D_{1}}+\sqrt{\log \log N} .
$$

Write $C$ for the absolute constant hiding in the above expression. We optimize $\eta$ by taking

$$
\eta^{-1}=2 C\left(\log \alpha^{-1}+\sqrt{\log D_{1}}+\sqrt{\log \log N}\right),
$$

and so we have derived a contradiction and must be in another of the above cases. By assumption we are not in the second case so we conclude that either

$$
\log N \ll \eta^{-2} \log \alpha^{-1}+\log ^{2} D_{1} \quad \text { or } \quad \log D_{1} \ll \eta^{-1} \log \alpha^{-1} .
$$

Inserting our choice of $\eta$ we get either

$$
\begin{aligned}
& \log N \ll \log ^{2} D_{1} \\
& \quad \text { or } \quad \log D_{1} \ll \log \alpha^{-1}\left(\log \alpha^{-1}+\sqrt{\log D_{1}}+\sqrt{\log \log N}\right) .
\end{aligned}
$$

CASE 2: $\left(D_{1}, D_{0}\right)$ is unexceptional. In this case we can proceed directly without the aid of Lemma 9.1. Let $\eta>0$ be a (new) parameter to be optimized later and let $P^{\prime}$ be an arithmetic progression such that $\alpha^{\prime} N^{\prime \prime} \eta^{2} d^{\prime-\eta}$ is maximal, where $\alpha^{\prime}$ is the relative density of $A$ on $P^{\prime}, N^{\prime}$ is the length of $P^{\prime}$ and $d^{\prime}$ is the common difference of $P^{\prime}$.

As before, in view of the maximality of $P^{\prime}$, we have

$$
\alpha \ll \alpha^{\prime}, \quad \log d^{\prime} \ll \eta^{-1} \log \alpha^{-1}
$$

and

$$
\log N=\log N^{\prime}+O\left(\eta^{-2} \log \alpha^{-1}\right) .
$$

Again, let $I^{\prime}:=\left\{1, \ldots, N^{\prime}\right\}$ and $A^{\prime}$ be the affine transformation of $A_{D} \cap P^{\prime}$ so that it lies in $I^{\prime}$. Apply Lemma 8.1 to get the following possibilities: either

(i) there is a progression $P^{\prime \prime}$ of common difference $d^{\prime \prime}=O\left(\alpha^{\prime-2}\right)$ and length at least $\left(c_{9} \alpha^{\prime} / d^{\prime} \log N^{\prime}\right)^{8} N^{\prime}$ with $\left|A^{\prime} \cap P^{\prime \prime}\right| \geq \alpha^{\prime}\left(1+c_{8}\right)\left|P^{\prime \prime}\right|$; or

(ii) $A^{\prime}-A^{\prime}$ contains a number of the form $(p-1) / d^{\prime}$ with $p$ a prime; or 
(iii) $N^{\prime} \leq O\left(\exp \left(c_{10} \log ^{2} D_{1}\right)\right)$ whence, by (9.5), $\log N \ll \eta^{-2} \log \alpha^{-1}+$ $\log ^{2} D_{1}$; or

(iv) $d^{\prime-1}=O\left(D_{0}^{-c_{10}} / \log ^{2} D_{1}\right)$ whence $d^{\prime} \gg D_{1}^{c_{10}^{2} / 4}$ and so, by (9.4), $\log D_{1} \ll \eta^{-1} \log \alpha^{-1}$; or

(v) $\alpha^{\prime}=O\left(D_{0}^{-c_{10}} / \log ^{2} D_{1}\right)$.

The analysis proceeds much as before and we conclude that either

(9.6) $\quad \log N \ll \log ^{2} D_{1} \quad$ or $\quad \log D_{1} \ll \log \alpha^{-1}\left(\log \alpha^{-1}+\sqrt{\log \log N}\right)$.

Write $C$ for the larger of the two constants hiding in the first inequalities in (9.3) and (9.6). We optimize $D_{1}$ by taking $\log N=2 C \log ^{2} D_{1}$ so that we never are in the first case of either. The result follows.

Acknowledgments. The second author would like to thank Ben Green.

\section{References}

[Dav00] H. Davenport, Multiplicative Number Theory, 3rd ed., Grad. Texts in Math. 74, Springer, New York, 2000.

[HB87] D. R. Heath-Brown, Integer sets containing no arithmetic progressions, J. London Math. Soc. (2) 35 (1987), 385-394.

[Luc07] J. Lucier, Difference sets and shifted primes, preprint, 2007.

[MV07] H. L. Montgomery and R. C. Vaughan, Multiplicative Number Theory I. Classical Theory, Cambridge Stud. Adv. Math. 97, Cambridge Univ. Press, Cambridge, 2007.

[PSS88] J. Pintz, W. L. Steiger and E. Szemerédi, On sets of natural numbers whose difference set contains no squares, J. London Math. Soc. (2) 37 (1988), 219-231.

[Ruz82] I. Z. Ruzsa, Uniform distribution, positive trigonometric polynomials and difference sets, in: Seminar on Number Theory, 1981/1982, Univ. Bordeaux I, Talence, 1982, exp. no. 18.

[Ruz84] -, On measures on intersectivity, Acta Math. Hungar. 43 (1984), 335-340.

[Sár78] A. Sárközy, On difference sets of sequences of integers. III, Acta Math. Acad. Sci. Hungar. 31 (1978), 355-386.

[Sze90] E. Szemerédi, Integer sets containing no arithmetic progressions, Acta Math. Hungar. 56 (1990), 155-158.

Alfréd Rényi Institute of Mathematics

Hungarian Academy of Sciences

Budapest, Pf. 127

H-1364 Hungary

E-mail: ruzsa@renyi.hu
Department of Pure Mathematics and Mathematical Statistics University of Cambridge Wilberforce Road

Cambridge CB3 0WA, England E-mail: t.sanders@dpmms.cam.ac.uk 